\title{
GASO 2-2013: Femenina de 23 años con síndrome pulmón-riñón (Síndrome de Goodpasture) en contexto de Lupus Eritematoso Sistémico
}
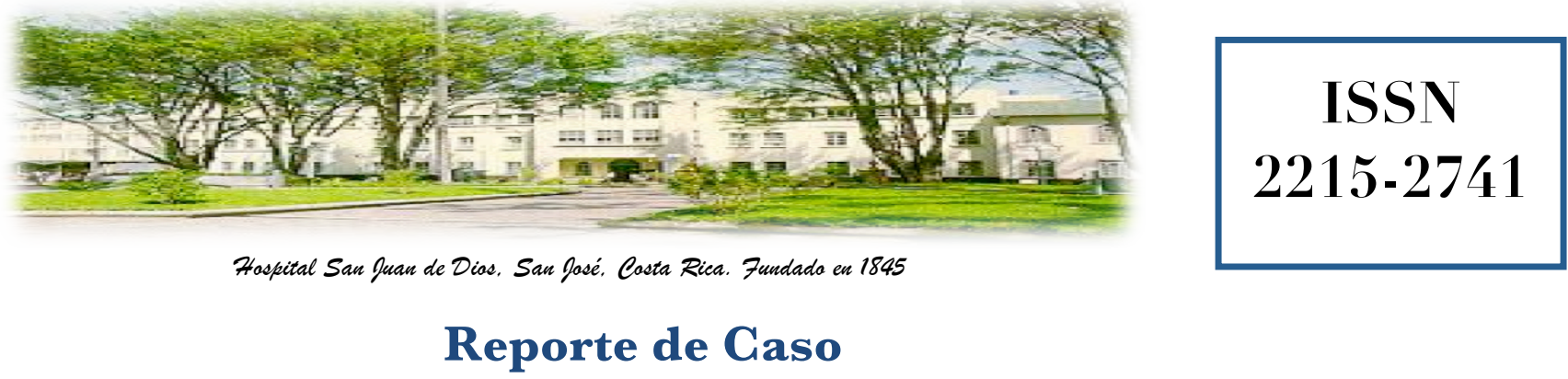

\section{Reporte de Gaso}

$\begin{array}{ll}\text { Recibido: } & 14 / 02 / 2013 \\ \text { Aceptado: } & 22 / 02 / 2013\end{array}$

Gabriel Torrealba Acosta ${ }^{1}$ Ignacio Salom Echeverría ${ }^{2}$ Julián Peña Varela ${ }^{3}$

\footnotetext{
${ }^{1}$ Médico Residente de Neurología, UCR-CENDEISSS. Hospital México. Profesor del Departamento de Anatomía, Escuela de Medicina UCR. Correo electrónico: doctortorrealba@gmail.com

${ }^{2}$ Médico Especialista en Medicina Interna. Asistente del Servicio de Medicina Interna. Hospital México.

${ }^{3}$ Médico Especialista en Medicina Interna. Jefe del Servicio de Medicina Interna, Hospital México.
}

\section{RESUMEN}

Paciente femenina de 23 años, hipotiroidea, con cuadro crónico de síntomas constitucionales, mialgias y artralgias; asociado a cuadro agudo de síntomas respiratorios, fiebre y dolor abdominal, acompañado por anemia microcítica hipocrómica con reticulocitosis, linfopenia y cardiomegalia. Se hace diagnóstico de de lupus eritematoso sistémico (LES), posterior a lo cual, la paciente desarrolla insuficiencia respiratoria y renal aguda asociada a hemorragia alveolar difusa, compatible con Síndrome de Good Pasture. En el presente caso se discute el síndrome riñónpulmón (Síndrome de Goodpasture) en el contexto de LES.

\section{PALABRAS CLAVE}

Lupus Eritematoso Sistémico. Hemorragia Alveolar Difusa. Síndrome de Good Pasture.

\begin{abstract}
A 23 year-old woman, with a previous diagnosis of hypothyroidism, was admitted to the hospital with a medical history of chronic constitutional symptoms, myalgias and arthralgias; and acute respiratory symptoms with fever and abdominal pain. Hypochromic microcytic anemia, lymphopenia and cardiomegaly on chest X-rays were demostrated. After the diagnosis of systemic lupus erythematous (SLE) she developed respiratory and renal failure with difusse alveolar hemorrage. In this case, the pulmonary-renal syndrome (Goodpasture's syndrome) within de context of SLE is described.
\end{abstract}

\section{KEY WORDS}

Systemic Lupus Erythematous. Diffuse Alveolar Hemorrhage. Goodpasture's Syndrome. 


\section{CASO CLÍNICO}

Femenina de 23 años con diagnóstico de hipotiroidismo de causa desconocida. Un embarazo previo, sin abortos y fecha de última menstruación de dos meses previos, con ciclos menstruales regulares. Sin antecedentes familiares conocidos. No otros antecedentes. Consultó por cuadro crónico (un año y medio de evolución) carracterizado por pérdida de peso, fatiga, malestar general, hiporexia y dolor muscular generalizado. En la valoración inicial se documentó anemia y ameritó trasfusión sanguínea (no se conoce estudios adicionales durante dicha intervención).

Agregó cuadro agudo respiratorio, con disnea en reposo que se agravaba con la actividad física, asociado a tos no productiva y dolor pleurítico. Luego sumó sensación febril de predominio nocturno, acompañada de diaforesis y dolor abdominal, sin nauseas, vómitos ni diarrea. No presentó síntomas urinarios.

Consultó por segunda ocasión al servicio de emergencias del Hospital México, donde se describió muy adelgazada, con fascies de crónicamente enferma, bradipsíquica, con respiración superficial y alopecia frontal. Se documentó dificultad en la apertura bucal y lesión aftosa en comisura labial derecha; cuello con adenopatías submandibulares bilaterales, pequeñas, móviles y levemente dolorosas. Ruidos cardíacos rítmicos sin soplos y campos pulmonares con murmullo vesicular disminuido en ambas bases, con escasas crepitaciones bibasales. Abdomen con resistencia muscular voluntaria y dolor a la palpación de hipocondrio y flanco derecho, sin masas ni visceromegalias palpables y con peristalsis presente. En miembros inferiores se observó petequias bilaterales y edema bipodal, sin ingurgitación yugular a $45^{\circ}$. Fuerza muscular y reflejos miotendinosos simétricos, no signo de Hoffmann ni Babinski, con marcha dificultosa pero sin ataxia ni lateropulsión.

En los exámenes de laboratorio iniciales (Cuadro 1) se destacó hiponatremia leve, función renal y hepática dentro de rangos normales, anemia microcítica-hipocrómica con reticulocitosis y leucopenia con linfopenia marcada, asociada a elevación de reactantes de fase aguda.

\section{Cuadro 1. Valores iniciales de laboratorio}

\begin{tabular}{|lc|}
\hline Variable & \multicolumn{1}{c|}{ Valor } \\
\hline Nitrogeno ureico & $21 \mathrm{mg} / \mathrm{dL}$ \\
\hline Creatinina & $0.8 \mathrm{mg} / \mathrm{dL}$ \\
\hline Sodio & $127.7 \mathrm{mEq} / \mathrm{L}$ \\
\hline Potasio & $3.61 \mathrm{mEq} / \mathrm{L}$ \\
\hline Calcio & $9.0 \mathrm{mEq} / \mathrm{L}$ \\
\hline FA & $60 \mathrm{UI} / \mathrm{L}$ \\
\hline GGT & $10 \mathrm{UI} / \mathrm{L}$ \\
\hline BT & $0.1 \mathrm{mg} / \mathrm{dL}$ \\
\hline AST & $18 \mathrm{UI} / \mathrm{L}$ \\
\hline ALT & $6 \mathrm{UI} / \mathrm{L}$ \\
\hline Hemoglobina & $6.3 \mathrm{~g} / \mathrm{dL}$ \\
\hline HCM & $24.6 \mathrm{pg}$ \\
\hline VCM & $79 \mathrm{fL}$ \\
\hline Retis (corregidos) & $3.4 \%$ \\
\hline Plaquetas & $322000 / \mathrm{mm}^{3}$ \\
\hline Leucocitos & $3800 / \mathrm{mm}^{3}$ \\
\hline PMN & $80 \%$ \\
\hline Linfocitos & $16 \%\left(608 / \mathrm{mm}^{3}\right)$ \\
\hline Bandas & $2 \%$ \\
\hline Albúmina & $2.0 \mathrm{~g} / \mathrm{dL}^{3}$ \\
\hline VES & $116 \mathrm{~mm} / \mathrm{h}$ \\
\hline PCR & $86.2 \mathrm{mg} / \mathrm{dL}^{2}$ \\
\hline
\end{tabular}

Fuente: revisión del expediente

En la radiografía de tórax inicial (Figura 1) se documentó cardiomegalia, con morfología "en bota" asociada a una consolidación en cuña en ángulo costodiafragmático derecho y cisuritis en pulmón derecho.

Figura 1. Radiografía AP de tórax inicial.

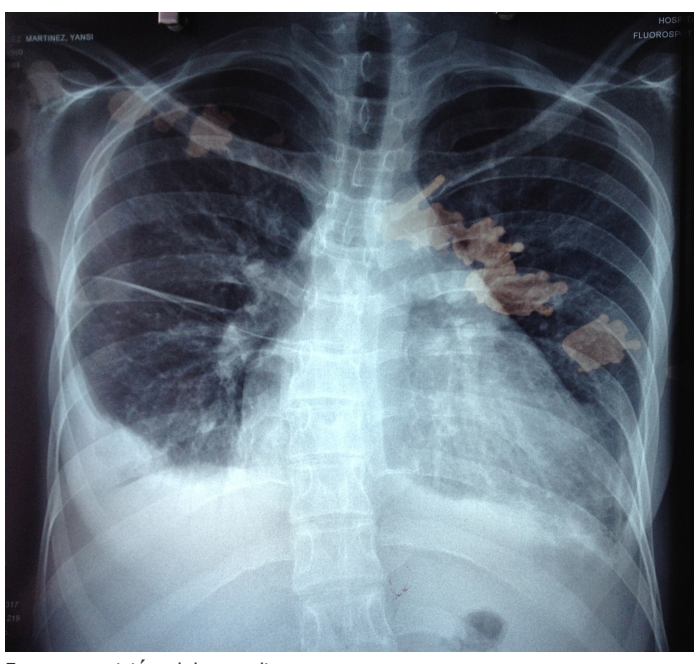

Fuente: revisión del expediente 


\section{Laboratorio y Gabinete}

TAC (Tomografía Axial Computarizada) de abdomen: hepatomegalia difusa. Focalización hipodensa sugerente de metástasis, incontables adenopatías intra y retroperitoneales, en unión esofagogástrica, en hilio esplénico, perigástricas, en hilio renal, paraaórticas, ilíacas comunes, internas y externas e inguinales. Dichas adenopatías con hilio graso, son consistentes con proceso inflamatorio o reactivo, con distribución sugerente de proceso linfoproliferativo. Esplenomegalia con múltiples lesiones hipodensas, aspecto clásico de infiltración por linfoma, con líquido libre pélvico y en ambas correderas paracólicas.

TAC de tórax: no se evidenció masas ni adenopatías sospechosas en mediastino, con estructuras vasculares conservadas, cámaras cardiacas sin alteraciones y pulmones con áreas de consolidación posterobasales de ambos lóbulos inferiores, asociado a derrame pleural bilateral de predominio derecho.

En este punto, el principal diagnóstico a descartar fue el linfoma; sin embargo, se realizó ultrasonido de abdomen que confirmó la hepatomegalia, el líquido libre en ambas correderas paracólicas y no describió las lesiones focales en hígado ni en bazo. Reportó además retroperitoneo ganglionar y vascular $\sin$ alteraciones.

Ecocardiograma: disfunción sistólica biventricular leve, con hipertrofia ventricular izquierda leve, derrame pericárdico leve sin datos de taponamiento.

Serologías por VIH (Virus de Inmunodeficiencia Humana) y VEB (Virus Ebstein Barr) negativas. La de CMV (Citomegalovirus) se reportó positiva tanto IgG como IgM.

Broncoscopía con lavado bronquioalveolar (LBA) documentó infección por Pseudomonas aureginosa, sin presencia de bacilos ácido alcohol resistentes. Se inició tratamiento con ceftazidime, con lo que la paciente presentó leve mejoría.

Perfil inmunológico: ANA, anticuerpos anti doble cadena de ADN, anti SSA, anti SSB y
ENA francamente positivos. Niveles de $\mathrm{C} 3$ y C4 disminuidos. Valoración por Reumatología: siete criterios diagnósticos para LES: úlceras orales, artritis, panserositis (derrame pleural, pericárdico y líquido peritoneal), alteración hematológica (anemia, linfopenia), anticuerpos presentes en LES previamente mencionados y ANA positivo. La alteración renal no se pudo objetivizar en orina de 24 horas, pero tenía examen general de orina con proteinuria importante.

\section{Evolución}

Se inició tratamiento para LES, con bolos de metilprednisolona y plaquinol, con lo cual la fiebre cayó y la paciente presentó mejoría clínica. Además, se inició anticoagulación con heparina de bajo peso molecular, dada la sospecha de que la consolidación en cuña que se observaba en la radiografía de tórax inicial (Figura 1), correspondiera a un infarto pulmonar, asociado a síndrome antifosfolípido. Por otro lado, debido al estado de desnutrición extremo que presentaba la paciente, se decidió iniciar nutrición parenteral y se colocó catéter venoso central yugular interno. En la radiografía de control posterior a la colocación de la vía central, se observó líquido ocupando el espacio pleural bilateral, pero de predominio derecho y engrosamiento pleural, por lo que se colocó tubo de tórax, a través del cual se obtuvo líquido abundante y sanguinolento.

Posteriormente, la paciente deterioró su función respiratoria y ameritó colocación de tubo endotraqueal. Al momento de la colocación, se evidenció franca hemoptisis.

En radiografías de control se observó infiltrados consolidados en parches en ambos campos pulmonares (Figura 2). Se realizó medición de Factor $\mathrm{X}$ activado, que se reportó en rangos de anticoagulación normal y se suspendió el tratamiento con heparina.

La paciente fue trasladada a la Unidad de Cuidados Intensivos (UCI), con diagnóstico de hemorragia alveolar difusa. Se inició soporte inotrópico y plasmaféresis.

Desarrolló insuficiencia renal aguda marcada con anuria, que ameritó soporte renal y luego de 
completar los ciclos de plasmaféresis se inició tratamiento con ciclofosfamida.

Figura 2. Radiografía AP de tórax control posterior a colocación de CVC.

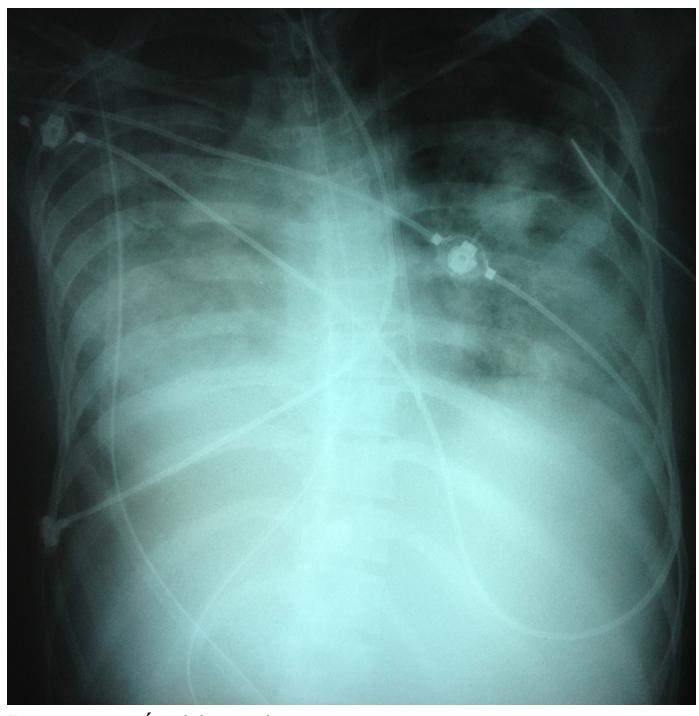

Fuente: Revisión del expediente.

Al final de un internamiento prolongado en la UCI, la paciente tuvo una evolución clínica satisfactoria, mejorando a los pocos días su función ventilatoria con cese de la hemorragia alveolar y aclaramiento de infiltrados en radiografías de tórax de control. Además, posterior al tratamiento con hemodiálisis mejoró su función renal.

\section{DISCUSIÓN}

El Síndrome Pulmón-Riñón incluye varias entidades, teniendo como grupo específico aquellas que se presentan con hemorragias alveolares difusas acompañadas de glomerulonefritis, frecuentemente de la variante rápidamente progresiva.

$\mathrm{Si}$ bien la hemorragia alveolar difusa en pacientes con LES ya había sido descrita en 1904 por Sir William Osler, a este conjunto de manifestaciones clínicas se le ha atribuido el nombre de Síndrome de Goodpasture en honor al teniente Ernest W. Goodpasture, quien describió por primera vez en 1919 la asociación de púrpura pulmonar y nefritis ${ }^{(1)}$.

Existen muchas causas que pueden dar lugar a este síndrome, por lo que se clasifican en inmunológicas y no inmunológicas. Las causas no imnunológicas incluyen infecciones (entre ellas CMV), algunos medicamentos, distrés respiratorio agudo, coagulopatías, radiación y uso de cocaína. Las causas inmunológicas más frecuentes incluyen las vasculitis asociadas a ANCA y los síndromes de anticuerpos anti membrana basal glomerular (anti GBM), cada uno de ellos tiene manifestaciones similares pero la fisiopatología y los hallazgos patológicos pueden $\operatorname{variar}^{(2)}$.

El LES es una de las causas inmunológicas que con menor frecuencia se asocia a este síndrome. El compromiso pulmonar se describe en un 0.5$5 \%$ de los casos, siendo la hemorragia alveolar difusa una forma frecuente de presentación, con hemoptisis franca en un $50 \%$ de las veces ${ }^{(1)}$. Tiene una mortalidad muy alta, que varía entre 70 y $90 \%$ y se presenta acompañada de nefritis en más del $90 \%$ de los casos. Además, es común encontrar la hemorragia alveolar asociada a anticuerpos antifosfolípido y existen casos reportados de ANCA y anti GBM presentes de forma concomitante $e^{(1,3)}$.

En los hallazgos histopatológicos de lesiones pulmonares en pacientes con LES, sólo $13 \%$ se presentan con capilaritis neutrofílica y otro $13 \%$ con hemorragia alveolar difusa, mientras que la mayoría no presentan otros cambios además de hemorragia alveolar, por lo que se le ha denominado "hemorragia pulmonar blanda". Con respecto a las lesiones vasculares renales, se han clasificado en lesiones no complicadas mediadas por depósito de complejos inmunes y la microangiopatía necrotizante no inflamatoria, también conocida como microangiopatía lúpica. Esta última es una forma de lesión capilar que es análoga a la observada en los capilares alveolares y ambas están mediadas por procesos de apoptosis casi con ausencia de inflamación en el vaso afectado. En estos casos de LES se identifica un proceso de apoptosis de células de paredes alveolares y de arteriolas renales, asociado con depósitos de complejos inmunes $^{(3,4)}$. 
Las manifestaciones clínicas de este síndrome en contexto de LES son variables, pero con frecuencia se establece el cuadro sin previo aviso. Es independiente también de la duración y evolución del LES, así como de la actividad de la enfermedad, por lo que puede ocurrir en cualquier momento de la evolución. También es independiente de haber recibido terapia inmunosupresora y puede ser la manifestación inicial del LES en un 10 a $20 \%$ de $\operatorname{los} \operatorname{casos}^{(4)}$. La manifestación clínica es inespecífica y con frecuencia se confunde con procesos infecciosos pulmonares, por lo que el diagnóstico temprano, si no se piensa en esta entidad, es poco frecuente.

Se puede manifestar con hipoxemia, disnea, tos o fiebre y en una tercera parte de los casos, se puede observar hemoptisis y disminución del hematocrito, siendo ambas condiciones las principales que favorecen un diagnóstico temprano. Como ya se mencionó antes, es frecuente el compromiso multiorgánico. El componente renal es el más prevalente de todos, manifestándose como insuficiencia renal, nefritis o síndrome nefrótico ${ }^{(2,4)}$.

Las manifestaciones radiológicas también son inespecíficas, presentándose con infiltrados alveolares e intersticiales difusos bilaterales y opacidades en parches; que con frecuencia se consideran de etiología infecciosa y así se retrasa el tratamiento específico. Por esta razón, otro de los factores que permite realizar un diagnóstico temprano es la falta de respuesta a antibioticoterapia, en comparación a una rápida resolución radiológica luego de iniciada la terapia con esteroides. En estos casos se sugiere además realizar broncoscopía con LBA, que permite corroborar sangrado intraalveolar $y$ hacer el diagnóstico diferencial con infección.

Sin embargo, es importante agregar que de un 30 a $60 \%$ de los pacientes presentan infección concomitante. El principal problema radica en enfocarse únicamente en la infección sin considerar la posibilidad de una hemorragia alveolar asociada, lo cual retrasa el diagnóstico y esta demora probablemente explique la alta mortalidad que tiene esta patología ${ }^{(4)}$.

$\mathrm{Si}$ bien esta entidad es independiente de la evolución y actividad de la enfermedad de fondo, existen ciertas condiciones que aumentan la probabilidad diagnóstica de esta patología.
Dentro de éstas se incluyen: altos niveles de anticuerpos anti ADN doble cadena, bajos niveles de complemento, puntaje de SLEDAI > 9, dosis altas de prednisona en 3 meses previos, nefritis, serositis, lupus neuropsiquiátrico e hipertensión pulmonar ${ }^{(4,5)}$.

En cuanto al manejo terapéutico, lo principal en el escenario agudo es un adecuado soporte ventilatorio además de mantener el conteo plaquetario mayor a 50.000 por $\mathrm{mm}^{3}$ y un INR de menos de 1.5. En caso de presentarse alguna coagulopatía asociada, es necesario considerar la transfusión de plaquetas y el aporte de vitamina $\mathrm{K}$ o plasma fresco congelada para su corrección. En casos refractarios, se ha sugerido la administración de de Factor VII recombinante activado, ya sea por vía sistémica o a través de broncoscopía, con reportes de casos en donde ha sido exitoso. También se ha utilizado ácido aminocaprocio como una opción para casos refractarios $^{(2)}$.

Finalmente, lo más importante es el manejo de la enfermedad subyacente, en este caso el LES. Aquí la terapia inicial es con esteroides en bolos seguido de la implementación de alguna terapia para alcanzar remisión de la enfermedad, en donde el tratamiento que ha demostrado aumento de sobrevida sigue siendo la ciclofosfamida. Sin embargo, debido a la toxicidad de su uso crónico, se ha estudiado otros tratamientos de mantenimiento, en donde la azatioprina y el metrotexate han demostrado eficacia similar.

El micofenolato de mofetilo, si bien es otra opción con un mejor perfil de seguridad, ha probado ser inferior a la azatioprina para alcanzar un adecuado mantenimiento de la remisión y es por ello que se ha dejado como tratamiento de segunda línea. En casos agudos y con poca respuesta a esteroides se puede utilizar plasmaféresis, no obstante los distintos estudios que han evaluado este tratamiento han arrojado resultados controversiales. En algunos pacientes refractarios se ha probado con rituximab y con inmunoglobulina, obteniendo efectos favorables en algunos $\operatorname{casos}^{(2,6-8)}$.

Se ha descrito algunas condiciones que aumentan el riesgo de mortalidad en estos pacientes que incluyen: la necesidad de ventilación mecánica asistida, las infecciones concomitantes y puntajes 
de SLEDAI altos y la principal causa de muerte en estos pacientes sigue siendo la falla respiratoria.

En cuanto al pronóstico, aquellos pacientes que sobreviven a la fase aguda tienen una sobrevida de un $75 \%$ a dos años, la mayoría recupera la función respiratoria y la función renal, siendo los más afectados aquellos que presentan este síndrome asociado a anticuerpos anti GBM. Algunos pacientes pueden evolucionar a fibrosis pulmonar $^{(9)}$. No obstante, en los pacientes que sobreviven a la fase aguda, posteriomente, la terapia inmunosupresora $\mathrm{y}$ especialmente la ciclofosfamida representa una importante causa añadida de morbimortalidad ${ }^{(10)}$.

\section{CONCLUSIONES}

El presente caso es para ilustrar una manifestación poco usual del LES, cuyo diagnóstico temprano es con frecuencia pasado por alto y que en este caso fue posible de identificar gracias a la colocación de un sello de tórax y la presencia de líquido sanguinolento en la sonda.

Si bien es cierto, a pesar de que no hay diagnóstico histopatológico del compromiso renal, ni se tiene reporte de ANCAs para clasificar el tipo de síndrome; dado el contexto del LES y si se toma en cuenta las condiciones que aumentan la probabilidad diagnóstica de esta entidad, en retrospectiva, se puede evidenciar que en efecto la paciente cumplió con la mayoría.

Es interesante agregar, que se presentó una infección concomitante por CMV, el cual está descrito como causante de lesión alveolar y podría ser que haya aportado algo a la clínica de esta paciente. Vale la pena destacar que en todo paciente con LES que se presenta con algún compromiso pulmonar es necesario tomar en consideración el síndrome pulmón-riñón, máxime cuando haya una mala respuesta a antibióticos. En estos casos, es necesario realizar mediciones seriadas de hemoglobina, con cultivos $y$ tomas de esputo e incluso broncoscopía con LBA, hasta descartar la presencia de hemorragia alveolar.
Si bien la paciente presentó una evolución satisfactoria de su cuadro, ubicándose en ese 10 a $30 \%$ de pacientes que sobreviven, se ha descrito recurrencias en pacientes sobrevivientes $y$ en aquellos pacientes en terapia de mantenimiento de la remisión, los efectos adversos se constituyen en una carga de morbimortalidad agregada importante, con muchos efectos secundarios y una muy poca adherencia.

\section{BIBLIOGRAFÍA}

1. Glassock R Khorashadi L Kushner Y. Case 32-2012: A 35-Year-Old Man with Respiratory and Renal Failure. N Engl J Med 2012;367:1540-1553.

2. Krause M Cartin-Ceba R Specks U Peikert T. Update on Diffuse Alveolar Hemorrhage and Pulmonary Vasculitis. Immunol Allergy Clin N Am 2012;32:587-600.

3. Hughson M He Z Henegar J McMurray R. Alveolar Hemorrhage and Renal Microangiopathy in Systemic Lupus Erythematosus: Immune Complex Small Vascular Injury With Apoptosis. Arch Pathol Lab Med 2001;125:475-483.

4. Shen M Zeng X Tian X et al. Diffuse alveolar hemorrhage in systemic lupus erythematosus: a retrospective study in China. Lupus 2010;19:1326-1330.

5. Kwok S Moon S Ju J et al. Lupus Around the World: Diffuse alveolar hemorrhage in systemic lupus erythematosus: risk factors and clinical outcome: results from affiliated hospitals of Catholic University of Korea. Lupus 2011;20:102-107.

6. Lee R D'Cruz D. Pulmonary renal vasculitis syndromes. Autoimmunity Reviews 2010;9:657-660 .

7. McCabe C Jones Q Nikolopoulou A et al. Pulmonary-renal syndromes: An update for respiratory physicians. Respiratory Medicine 2011;105:1413-1421.

8. Papiris S Manali E Kalomenidis I et al. Bench-to-bedside review: Pulmonary-renal syndromes - an update for the intensivist. Critical Care 2007;11(3):213-224.

9. Specks U. Diffuse alveolar hemorrhage syndromes. Rheumatol 2001;13:12-17.

10. Gallagher H Kwan J Jayne D. Pulmonary Renal Syndrome: A 4-Year, Single-Center 
Experience. American Journal of Kidney Diseases 2002;39(1):42-47. 